\title{
EFFECT OF POTASSIUM BUDGET ON EVOLUTION OF SOIL POTASSIUM IN DIFFERENT CROP SEQUENCES AND SITE CONDITIONS
}

\author{
MIKULÁŠ MADARAS
}

Crop Research Institute, Prague

MADARAS, M.: Effect of potassium budget on evolution of soil potassium in different crop sequences and site conditions. Agriculture (Pol’nohospodárstvo), vol. 60, 2014, no. 4, pp. 121-131.

\begin{abstract}
The influence of six different crop sequences on the dynamics of topsoil available and fixed potassium $\left(\mathrm{K}_{\text {avail }}\right.$ and $\mathrm{K}_{\mathrm{fix}}$ ) was studied within a 4 -year period at 6 sites of different soil and climatic conditions. The highest $\mathrm{K}$ offtakes were recorded for 3-year alfalfa/ clover cropping followed by winter wheat $(-151 \mathrm{~kg} \mathrm{~K} / \mathrm{ha} / \mathrm{year})$ and for the crop sequence including one year of sugar beet / potato $\left(-124 \mathrm{~kg} \mathrm{~K} / \mathrm{ha} /\right.$ year). $\mathrm{K}_{\text {avail }}$ and $\mathrm{K}_{\text {fix }}$ were significantly decreased especially at crop sequence with alfalfa / clover cropping, compared to other crop sequences. At three sites of lower Kfix content (340-830 mg K/ $\mathrm{kg}$ ), differences in $\mathrm{K}_{\text {avail }}$ and $\mathrm{K}_{\text {fix }}$ between crop sequences were
\end{abstract}

more frequent, whereas almost no significant differences between treatments were observed at sites of higher $\mathrm{K}_{\text {fix }}$ content $(1,000-1,380 \mathrm{mg} \mathrm{K} / \mathrm{kg})$. Changes of both $\mathrm{K}$ forms and $\mathrm{K}$ budget correlated significantly at sites of lower $\mathrm{K}_{\text {fix }}$ content. At these sites, $\mathrm{K}_{\text {avail }}$ decreased at the rate of $0.045 \mathrm{mg} \mathrm{K}$ per $1 \mathrm{~kg} / \mathrm{ha}$ of $\mathrm{K}$ offtake; the rate of $\mathrm{K}_{\text {fix }}$ decrease was $0.059 \mathrm{mg} \mathrm{K}$ per $1 \mathrm{~kg} / \mathrm{ha}$ of $\mathrm{K}$ offtake. At sites of higher $\mathrm{K}_{\text {fix }}$, no significant relation between $\mathrm{K}$ forms and $\mathrm{K}$ budget was observed. The results show an importance of $\mathrm{K}_{\text {fix }}$ as a $\mathrm{K}$ source in intensive agricultural systems with low or no $\mathrm{K}$ inputs.

Key words: fertilisation, nutrients, budget, balance, non-exchangeable potassium

Although the use of potassium $(\mathrm{K})$ fertilisers increased by $25 \%$ since 1980 in global scale (Zörb et al. 2014), their application decreased dramatically in northern and western Europe (Öborn et al. 2005). In the Czech Republic, the use of $\mathrm{K}$ fertilisers dropped after 1989 from the level of ca. $70 \mathrm{~kg} \mathrm{~K} 2 \mathrm{O} / \mathrm{ha}$ to the level as low as ca. $10 \mathrm{~kg} \mathrm{~K}_{2} \mathrm{O} / \mathrm{ha}$ (Grzebisz et al. 2010). Recent $\mathrm{K}$ input to agricultural soils is at the level of $5 \mathrm{~kg} \mathrm{~K} / \mathrm{ha}$ in the form of mineral fertilisers. In addition, $18 \mathrm{~kg} \mathrm{~K} / \mathrm{ha}$ is applied in the form of manures (Anonymous 2013). Overall field K offtake by harvested products is estimated to $71 \mathrm{~kg}$ $\mathrm{K} /$ ha on average (Klír et al. 2008); the exact offtake depends on the selection of crops and their yields.

With insufficient external $\mathrm{K}$ input, plant demand for $\mathrm{K}$ is fulfilled by previously bound fertiliser $\mathrm{K}$ and by internal soil $\mathrm{K}$ sources. $\mathrm{K}$ content in soils ranges from 0.4 to $30 \mathrm{~g} / \mathrm{kg}$. Only $2-10 \%$ of soil $\mathrm{K}$ is available to plants, the main pool is present as hardly available structural $\mathrm{K}$ in K-bearing feldspars and layer silicates (Huang 2005; Zörb et al. 2014). The term "available $\mathrm{K}$ " $\left(\mathrm{K}_{\text {avai }}\right)$ is often used in agronomy for the pool consisting predominantly of water-soluble and exchangeable K. Non-exchangeable K is another significant pool available to plants, too. This pool, called also reserve $\mathrm{K}$, interlayer $\mathrm{K}$ or fixed $\mathrm{K}$ $\left(\mathrm{K}_{\mathrm{fix}}\right)$, is neither bonded covalently within the crystal structures of mineral particles nor is exchangeable. Its relation to exchangeable and water-soluble $\mathrm{K}$ is weak (Torma 1999). $\mathrm{K}_{\text {fix }}$ is moderately to less available to plants, depending on soil properties (Martin \& Sparks 1985). A release of $\mathrm{K}_{\text {fix }}$ to easily available forms is forced by plant $\mathrm{K}$ removal, microbial activity and by $\mathrm{K}$ leaching (Moritsuka et al. 2004; Martin \& Sparks 1985). The measurement of $\mathrm{K}_{\text {fix }}$ is often suggested to improve prediction of $\mathrm{K}$ availability 
to plants (Neuberg 1980; Rees 2013; Edmeades et al. 2014), but in the Czech Republic these analyses were not included within the common testing of agricultural soils.

As it is known from long-term field fertilisation experiments, non-use of $\mathrm{K}$ fertilisers leads first to the decrease of $\mathrm{K}_{\text {avail }}$ and afterwards to the stabilisation of $\mathrm{K}_{\text {avail }}$ at a certain level ranging from 30 to $120 \mathrm{mg} \mathrm{K} / \mathrm{kg}$ (Blake et al. 1999). At this stage, further $\mathrm{K}$ uptake is compensated by $\mathrm{K}$ release from $\mathrm{K}_{\text {fix }}$. Our previous research concerning long-term experiments with graduated $\mathrm{K}$ input indicated at some sites that the more negative is $\mathrm{K}$ budget, the more intensive is depletion of both $\mathrm{K}_{\text {avail }}$ and $\mathrm{K}_{\text {fix }}$ (Madaras et al. 2014). The decrease of $\mathrm{K}_{\text {fix }}$ can have the same magnitude as the decrease of $\mathrm{K}_{\text {avail }}$. In some cases, the whole $\mathrm{K}$ offtake can originate from $\mathrm{K}_{\text {fix }}$ pool (Øgaard \& Hansen 2010).

The experimental design of graduated fertiliser rates is common in short-term and long-term field fertilisation experiments, while other factors such as crop sequence are usually uniform for all trial plots (Blake et al. 1999; Øgaard \& Hansen 2010; Hejcman et al. 2013; Madaras et al. 2014). However, crops differ in their nutrient uptake, which consequently have an influence on the nutrient budget. Some crops have also specific mechanisms or adaptations by which they can increase the uptake from $\mathrm{K}_{\text {fix }}$ (Rengel \& Damon 2008). To study the influence of different crop plants on short-term soil K changes in agricultural systems without $\mathrm{K}$ input, we established the 4-year field experiment where different crop sequences were included as treatments. The objective of the research presented in this paper is to evaluate the influence of selected typical crop plants and their sequences on evolution of soil K.

\section{MATERIAL AND METHODS}

\section{Experimental design}

Field small-plot experiment was established at 6 sites with different climatic and soil conditions (Table 1). Sites were selected from the research station network of the Crop Research Institute, Prague. At each site, the experiment consisted of 24 plots of the size $3 \times 10 \mathrm{~m}$. An experimental design included 6 treatments, differing by the sequence of typical crop plants (Table 2). Treatments were replicated four times at each site. The same crop varieties and the same fertilisation scheme were used at all sites, with respect to particular crop production region. At colder sites, sugar beet was replaced by potato and alfalfa was replaced by clover. Treatments A, $\mathrm{B}, \mathrm{C}$ and $\mathrm{D}$ differed only by the crop in the $2^{\text {nd }}$ year of the experiment. Treatment E represented fodder production and included crops with high $\mathrm{K}$ uptake (alfalfa / clover). Treatment F was soil without vegetation cover within the first 3 years, weeds were suppressed chemically or mechanically when it was necessary. Exceptions of the uniform design were (1) the use of mustard or spring rapeseed instead of winter rapeseed after damage in winter 2010/2011 and (2) cropping of winter triticale instead of winter

$$
\mathrm{T} \text { a }
$$

Basic characteristics of experimental sites and soils

\begin{tabular}{|l|c|c|c|c|c|c|c|c|c|c|c|}
\hline Site & Soil type & $\begin{array}{c}\mathrm{A} \\
{[\mathrm{m}]}\end{array}$ & $\begin{array}{c}\mathrm{T} \\
{\left[{ }^{\circ} \mathrm{C}\right]}\end{array}$ & $\begin{array}{c}\mathrm{R} \\
{[\mathrm{mm}]}\end{array}$ & $\mathrm{Reg}$ & $\begin{array}{c}\mathrm{Clay} \\
{[\%]}\end{array}$ & $\begin{array}{c}\mathrm{C}_{\mathrm{ox}} \\
{[\%]}\end{array}$ & $\begin{array}{c}\mathrm{pH} \\
(\mathrm{KCl})\end{array}$ & \multicolumn{4}{|c|}{$\mathrm{K}_{\text {avail }}$} & $\mathrm{K}_{\text {fix }}$ & $\mathrm{P}_{\text {avail }}$ \\
\hline Ivanovice na Hané & Chernozem & 220 & 9.2 & 548 & $\mathrm{SB}$ & 24 & 2.2 & 6.5 & 186 & 1,050 & 177 \\
Hněvčeves (HK) & Luvisol & 265 & 8.1 & 597 & $\mathrm{SB}$ & 14 & 1.2 & 6.4 & 173 & 800 & 73 \\
Kostelec n. Orlicí & Luvisol & 290 & 8.0 & 696 & $\mathrm{SB}$ & 16 & 1.1 & 6.3 & 145 & 500 & 62 \\
Prague (Ruzyně) & Luvisol & 345 & 7.9 & 472 & $\mathrm{SB}$ & 19 & 1.5 & 6.8 & 187 & 1,010 & 62 \\
Pernolec (Tachov) & Cambisol & 530 & 7.1 & 559 & POT & 9 & 1.1 & 6 & 99 & 1,350 & 71 \\
Vysoké n. Jizerou & Cambisol & 670 & 6.0 & 1,000 & FOD & 13 & 2.1 & 5.5 & 207 & 350 & 12 \\
\hline
\end{tabular}

$\mathrm{A}$ - altitude, T - mean air temperature, $\mathrm{R}$ - annual sum of rainfall, Reg - production regions ( $\mathrm{SB}$ - sugar beet, POT - potato, FOD-fodder crop), Clay - fraction $<0.002 \mathrm{~mm}, \mathrm{~K}_{\text {avail }}$ and $\mathrm{P}_{\text {avail }}$ - available nutrients (Mehlich III extraction), $\mathrm{K}_{\text {fix }}-$ fixed $\mathrm{K}$ 
wheat in site Vysoké. Overview of the weather conditions during the experiment is presented in Figure 1 and Table 3.

\section{Sampling and analyses}

Plant main product and by-products were harvested each year. Harvested material was weighed at actual moisture. Representative samples of the plant material were taken for further laboratory analyses, which includes determination of moisture content (drying at $105^{\circ} \mathrm{C}$ until a constant weight) and total $\mathrm{K}$ content (total microwave digestion). $\mathrm{K}$ offtake by harvested material was expressed in $\mathrm{kg} / \mathrm{ha}$. Because none $\mathrm{K}$ was applied in fetrilisers during the experiment, $\mathrm{K}$ offtake is equal to the $\mathrm{K}$ budget.
Soil samples of the plough horizon $(0-20 \mathrm{~cm})$ were taken from each plot after the harvest of the last crop. Each sample consisted of 5-8 subsamples. Samples were dried at room temperature and sieved through $2 \mathrm{~mm}$ mesh. Two pools of soil $\mathrm{K}$ were analyzed. $\mathrm{K}_{\text {avail }}$ was extracted by the Mehlich 3 method (Mehlich 1984). $\mathrm{K}_{\text {fix }}$ was calculated as $\mathrm{K}$ extracted by $1 \mathrm{~mol} / 1 \mathrm{HCl}(1: 10 \mathrm{w} / \mathrm{v}$, incubation 20 h at $50^{\circ} \mathrm{C}$; adapted from Scheffer \& Schachtschabel 1976) subtracted by $K_{\text {avail }}$. Extracts were analyzed twice by AAS GBC 908AA. Statistical evaluation of the results was performed using the STATISTICA 12 software (Statsoft)

T

Crop sequences and fertilisation [kg nutrient/ha]

\begin{tabular}{|c|c|c|c|c|c|c|c|c|c|c|}
\hline \multirow{2}{*}{$\begin{array}{c}\text { Sequence } \\
\text { code }\end{array}$} & \multicolumn{2}{|c|}{2010} & \multicolumn{2}{c|}{2011} & \multicolumn{2}{|c|}{2012} & \multicolumn{3}{|c|}{2013} \\
\cline { 2 - 10 } & Crop & N1+N2 & Crop & N1+N2 & Crop & N1+N2 & P & Crop & N1+N2 & P \\
\hline A & ww & $50+50$ & ww & $40+60$ & bar & $50+50$ & 30 & ww & $40+60$ & 40 \\
B & ww & $50+50$ & pea & $0+30$ & bar & $50+50$ & 30 & ww & $40+60$ & 40 \\
C & ww & $50+50$ & sb / pot* & $60+40$ & bar & $50+50$ & 30 & ww & $40+60$ & 40 \\
$\mathrm{D}$ & ww & $50+50$ & rap / mus* & $40+30$ & bar & $50+50$ & 30 & ww & $40+60$ & 40 \\
E & alf / clo* & 0 & alf / clo* & 0 & alf / clo* & 0 & 0 & ww & $40+60$ & 40 \\
F & fallow & 0 & fallow & 0 & fallow & 0 & 0 & ww & $40+60$ & 40 \\
\hline
\end{tabular}

ww - winter wheat (triticale was used instead of winter wheat in all cases at Vysoké nad Jizerou), alf / clo - alfalfa / clover, sb / pot - sugar beet / potato, rap / mus - rapeseed / mustard, bar - spring barley

* - latter crop was used in Pernolec and Vysoké nad Jizerou

$\begin{array}{lllllllllll}\mathrm{T} & \mathrm{a} & \mathrm{b} & 1 & \mathrm{e} & 3\end{array}$

Overview of the weather conditions during the experiment

\begin{tabular}{|c|c|c|c|c|c|c|c|c|c|c|c|c|c|c|c|c|}
\hline \multirow{3}{*}{ Site } & \multicolumn{4}{|c|}{2010} & \multicolumn{4}{|c|}{2011} & \multicolumn{4}{|c|}{2012} & \multicolumn{4}{|c|}{2013} \\
\hline & \multicolumn{2}{|c|}{ I-XII } & \multicolumn{2}{|c|}{ IV-IX } & \multicolumn{2}{|c|}{ I-XII } & \multicolumn{2}{|c|}{ IV-IX } & \multicolumn{2}{|c|}{ I-XII } & \multicolumn{2}{|c|}{ IV-IX } & \multicolumn{2}{|c|}{ I-XII } & \multicolumn{2}{|c|}{ IV-IX } \\
\hline & $\mathrm{R}$ & $\mathrm{T}$ & $\mathrm{R}$ & $\mathrm{T}$ & $\mathrm{R}$ & $\mathrm{T}$ & $\mathrm{R}$ & $\mathrm{T}$ & $\mathrm{R}$ & $\mathrm{T}$ & $\mathrm{R}$ & $\mathrm{T}$ & $\mathrm{R}$ & $\mathrm{T}$ & $\mathrm{R}$ & $\mathrm{T}$ \\
\hline Ivanovice & 826 & 8.4 & 637 & 15.6 & 438 & 9.5 & 350 & 16.4 & 482 & 9.6 & 331 & 16.7 & 551 & 9.2 & 379 & 15.7 \\
\hline Hněvčeves & 792 & 8.4 & 591 & 15.6 & 552 & 9.4 & 361 & 16.0 & 772 & 9.1 & 518 & 15.9 & 615 & 8.9 & 424 & 15.3 \\
\hline Kostelec & 900 & 8.2 & 696 & 14.9 & 672 & 9.3 & 508 & 15.7 & 656 & 9.0 & 415 & 15.7 & 680 & 8.8 & 465 & 15.0 \\
\hline Prague & 679 & 8.9 & 531 & 15.4 & 605 & 9.6 & 437 & 16.2 & 563 & 9.8 & 437 & 16.3 & 731 & 8.3 & 559 & 15.3 \\
\hline Pernolec & 693 & 7.1 & 382 & 13.9 & 554 & 8.7 & 361 & 15.0 & 459 & 8.3 & 278 & 14.6 & 554 & 7.7 & 372 & 13.9 \\
\hline Vysoké & 1,231 & 5.8 & 733 & 12.8 & 984 & 7.3 & 598 & 13.7 & 1,127 & 6.2 & 529 & 13.7 & 969 & 6.6 & 534 & 12.7 \\
\hline
\end{tabular}

$\mathrm{R}$ - annual sum of rainfall, $\mathrm{T}$ - mean air temperature 


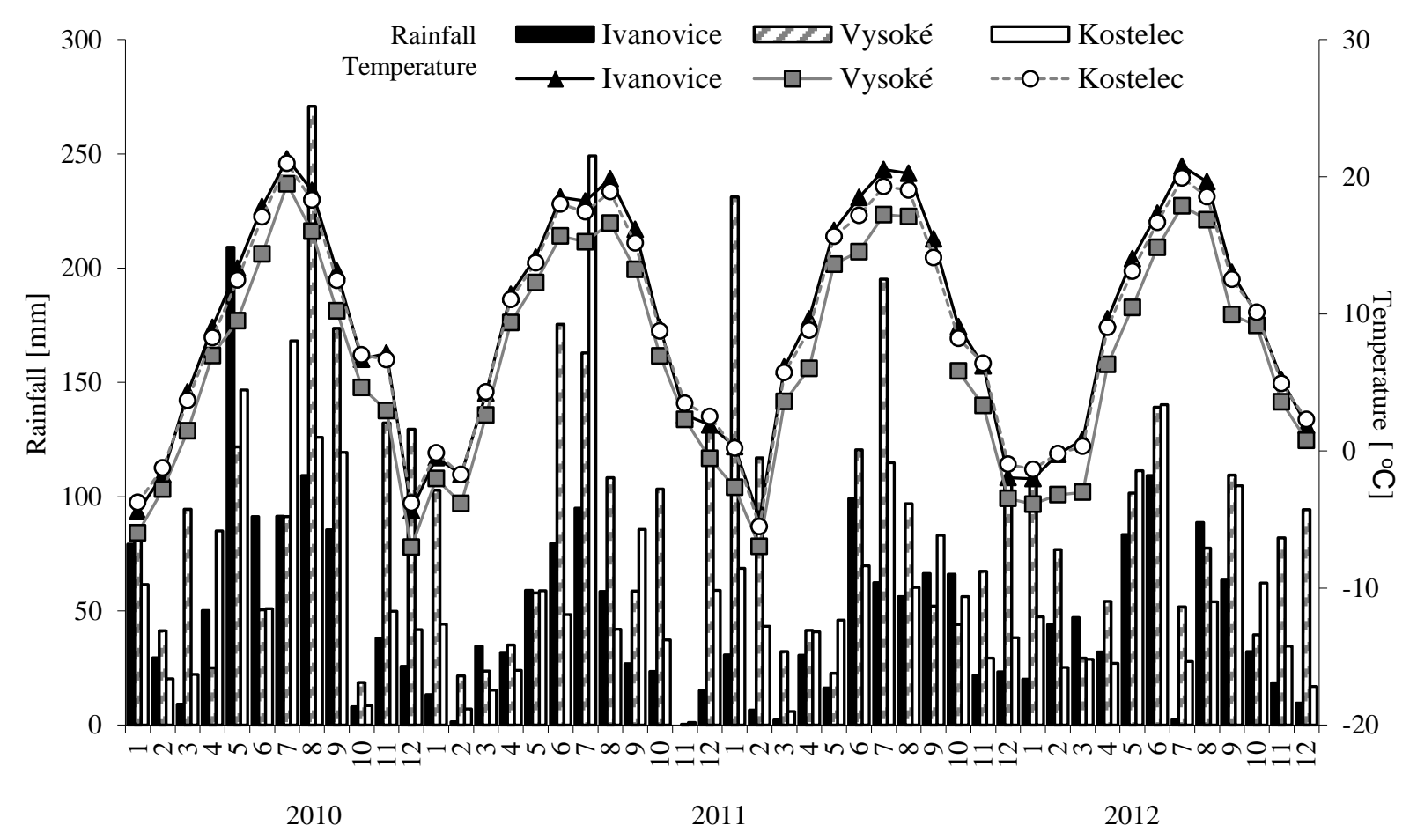

Figure 1. Overview of the weather conditions during the experiment (selected sites)

\section{RESULTS}

\section{Yields and K uptake}

Crop yields differed widely among experimental sites (Table 4). On average, they reached or exceeded average yields in the Czech Republic in particular years. The only exception was very low yield of rapeseed in 2011 due to the unfavourable late-winter and spring weather conditions at sites Hněvčeves, Prague and Ivanovice. High intensity of weed attack (e.g. Apera spica-venti) was one of the main reasons of low yields of winter wheat in Pernolec. Low rainfall caused decreased alfalfa yields in Ivanovice, especially in 2012.

Calculated $\mathrm{K}$ uptake by crops is shown in Table 5. The highest annual uptake $(327 \mathrm{~kg} \mathrm{~K} /$ ha on average in 2011) was recorded for sugar beet / potato cropping, with maximum of $415 \mathrm{~kg}$ $\mathrm{K} / \mathrm{ha}$ in Hněvčeves. By-products (leaves of sugar beet, stems and leaves of potato) accounted for $56 \%$ of $\mathrm{K}$ uptake. The second largest annual $\mathrm{K}$ uptake was recorded for alfalfa / clover during the $2^{\text {nd }}$ and $3^{\text {rd }}$ year of the experiment. On average, $244 \mathrm{~kg} \mathrm{~K} /$ ha was taken up annually by three cuts of hay. The lowest uptake was recorded for spring barley and for rapeseed. In the latter case, low uptake was caused both by low yield and by naturally low $\mathrm{K}$ concentration in the rapeseed biomass. Average annual $\mathrm{K}$ uptake by winter wheat was $59 \mathrm{~kg} \mathrm{~K} / \mathrm{ha}$; $41 \%$ of it accounted for grain. Triticale, as a replacement of winter wheat in Vysoké, showed more than 2-times higher $\mathrm{K}$ uptake compared to winter wheat because of higher straw $\mathrm{K}$ content $(1.1 \% \mathrm{~K}$ in triticale straw, $0.6 \% \mathrm{~K}$ in winter wheat straw).

The crop variations in $\mathrm{K}$ uptake are reflected in average $\mathrm{K}$ budgets for treatments. The most negative K budget was calculated for treatment E $(-184 \mathrm{~kg}$ $\mathrm{K} / \mathrm{ha} /$ year; average of 2011, 2012 and 2013), which was followed by the treatment C $(-150 \mathrm{~kg} \mathrm{~K} / \mathrm{ha} / \mathrm{year})$. In order of increasing $\mathrm{K}$ budget, next crop sequences were B $(-59 \mathrm{~kg} \mathrm{~K} / \mathrm{ha} /$ year $), A(-54 \mathrm{~kg}$ $\mathrm{K} / \mathrm{ha} /$ year), D ( $-53 \mathrm{~kg} \mathrm{~K} / \mathrm{ha} /$ year) and $\mathrm{F}(-26 \mathrm{~kg}$ $\mathrm{K} /$ ha/year; here only $\mathrm{K}$ uptake by winter wheat in 2013 was accounted). 

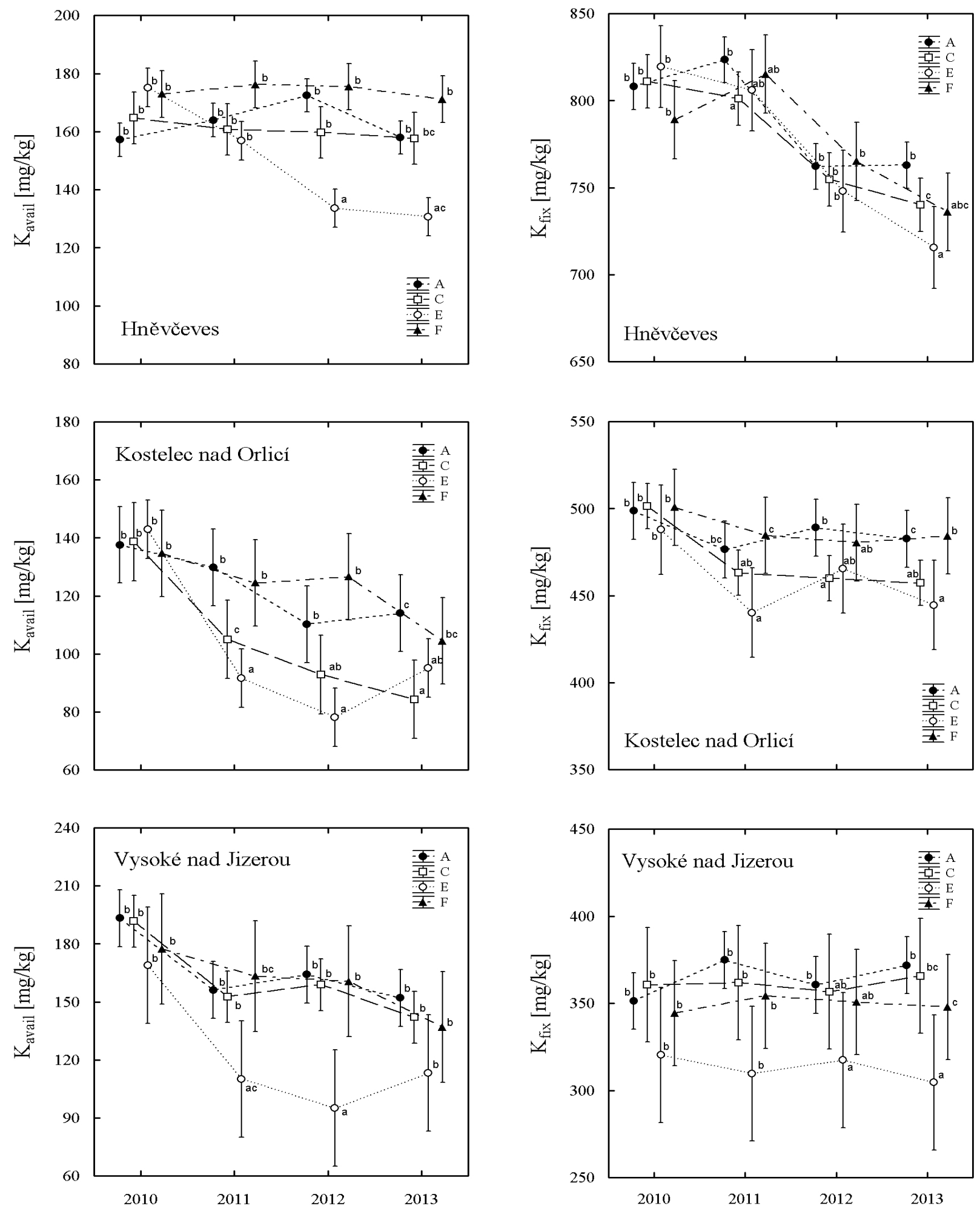

Figure 2. Available $\mathrm{K}$ contents at sites of fixed $\mathrm{K}<1,000 \mathrm{mg}$ $\mathrm{K} / \mathrm{kg}$ (arithmetic mean and standard deviation). In particular years, significant differences $(P<0.05)$ between treatments A, C, E and F are marked by different letters.

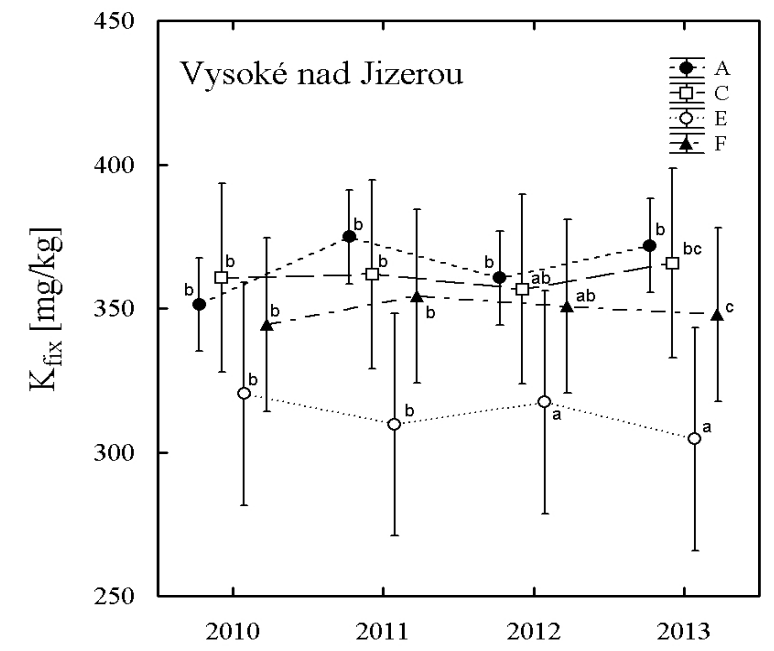

Figure 3. Fixed $\mathrm{K}$ contents at sites of fixed $\mathrm{K}<1,000 \mathrm{mg}$ $\mathrm{K} / \mathrm{kg}$ (arithmetic means and standard deviation). In particular years, significant differences $(P<0.05)$ between treatments are marked by different letters. 


\section{Soil available potassium}

Remarkable changes of $\mathrm{K}_{\text {avail }}$ were observed especially in Vysoké, Kostelec and Hněvčeves (Figure 2). At these 3 sites, $\mathrm{K}_{\text {avail }}$ of the treatment E was clearly different from other treatments. In Hněvčeves, $\mathrm{K}_{\text {avail }}$ at crop sequence $\mathrm{E}$ was significantly lower than at other crop sequences in years 2012 and $2013(P<0.01$ for almost all Student tests between treatments); however there were no significant differences among other crop sequences. Dur- ing the experiment, $\mathrm{K}_{\text {avail }}$ remained approximately at the same level, with exception of the continuous decrease by alfalfa cropping within 2010 and 2012 at treatment $\mathrm{E}$.

In Kostelec, a significant decrease of $\mathrm{K}_{\text {avail }}$ was observed for all treatments between 2010 and 2013 $(P<0.001)$. Since 2011, $\mathrm{K}_{\text {avail }}$ of treatments $\mathrm{C}$ and $\mathrm{E}$ was lower than that of other treatments $(P<0.05)$. In 2013, a slight increase of $\mathrm{K}_{\text {avail }}$ was observed for treatment E compared to the contents in $2012(P=0.01)$.

$\mathrm{T}$ a

Overview of crop yields at experimental sites in main product (MP) and by-product (BP) [t/ha].

Moisture: cereals / rapeseed / pea 14\%; sugar beet / potato actual moisture at harvest; alfalfa and clover - yields in dry matter. In brackets - standard deviations.

\begin{tabular}{|c|c|c|c|c|c|c|c|c|c|c|c|}
\hline \multirow{2}{*}{\multicolumn{2}{|c|}{ Site }} & \multicolumn{2}{|c|}{2010} & \multicolumn{5}{|c|}{2011} & \multicolumn{2}{|c|}{2012} & \multirow{2}{*}{$\frac{2013}{w W}$} \\
\hline & & ww & alf / clo* & ww & pea & $\mathrm{sb} /$ pot $^{*}$ & $\mathrm{rap} / \mathrm{mus}$ & alf / clo* & bar & alf / clo* & \\
\hline \multirow{4}{*}{ Ivanovice } & & 6.4 & 2.7 & 7.4 & 4.0 & 61 & 0.9 & 7.4 & 3.5 & 3.2 & 7.6 \\
\hline & $\mathrm{MP}$ & $(1.0)$ & $(0.6)$ & $(0.5)$ & $(0.7)$ & (21) & $(0.2)$ & $(0.5)$ & $(0.4)$ & $(0.2)$ & $(0.4)$ \\
\hline & DD & 4.0 & & 7.9 & 3.5 & 31 & 2.2 & & 3.9 & & 9.3 \\
\hline & BP & $(0.7)$ & - & $(0.8)$ & (0.6) & & $(0.4)$ & - & (0.6) & & (0.6) \\
\hline \multirow{4}{*}{ Hněvčeves } & & 10.2 & 3.3 & 11.1 & 4.5 & 89 & 1.0 & 15.9 & 6.3 & 18.3 & 6.6 \\
\hline & MP & $(0.5)$ & $(0.5)$ & $(0.3)$ & $(0.1)$ & (9.7) & $(0.1)$ & (2.1) & $(0.5)$ & (1.1) & (1.4) \\
\hline & & 11.9 & & 8.4 & 4.1 & 45 & 2.0 & & 6.4 & & 7.4 \\
\hline & BP & $(0.9)$ & - & $(0.5)$ & $(0.1)$ & & $(0.4)$ & - & (1.7) & - & (1.6) \\
\hline \multirow{4}{*}{ Kostelec } & & 8.3 & 4.0 & 7.0 & 2.6 & 99 & 3.3 & 9.1 & 6.6 & 9.3 & 10.6 \\
\hline & & $(0.5)$ & $(0.5)$ & $(0.8)$ & $(0.5)$ & (6.2) & $(0.1)$ & (1.9) & $(0.7)$ & $(0.3)$ & $(0.8)$ \\
\hline & & 4.4 & & 3.1 & 2.3 & 50 & 4.2 & & 3.0 & & 9.1 \\
\hline & BP & $(0.2)$ & - & $(0.2)$ & $(0.4)$ & & $(0.2)$ & - & $(0.3)$ & - & $(0.7)$ \\
\hline \multirow{4}{*}{ Prague } & & 7.6 & 5.7 & 5.6 & 2.9 & 82 & 0.7 & 19.7 & 3.2 & 15.3 & 10.7 \\
\hline & $\mathrm{MP}$ & $(0.5)$ & (1.0) & $(0.2)$ & $(0.0)$ & (11.5) & $(0.1)$ & (1.7) & $(0.5)$ & (1.8) & $(0.7)$ \\
\hline & & 3.8 & & 2.1 & 2.6 & 41 & 2.0 & & 2.1 & & 5.4 \\
\hline & BP & $(0.4)$ & - & $(0.3)$ & $(0.1)$ & & $(0.3)$ & - & $(0.3)$ & & $(0.5)$ \\
\hline \multirow{4}{*}{ Pernolec } & & 2.2 & 1.0 & 2.9 & 2.7 & 26.7 & 1.4 & 13.2 & 4.1 & 11.4 & 4.1 \\
\hline & $\mathrm{MP}$ & $(0.5)$ & $(0.2)$ & $(0.2)$ & $(0.2)$ & $(0.7)$ & $(0.1)$ & $(0.4)$ & $(0.4)$ & $(0.2)$ & (1.0) \\
\hline & & 1.7 & & 2.3 & 2.5 & & & & 2.2 & & 3.2 \\
\hline & BP & $(0.4)$ & - & $(0.2)$ & (0.3) & - & - & - & $(0.2)$ & - & $(0.8)$ \\
\hline \multirow{4}{*}{ Vysoké } & & 8.4 & 3.1 & 6.3 & 6.3 & 43 & 4.8 & 12.0 & 5.9 & 9.8 & 6.3 \\
\hline & $\mathrm{MP}$ & $(0.3)$ & $(0.4)$ & $(0.3)$ & $(0.3)$ & (1.0) & $(0.4)$ & $(0.7)$ & $(0.3)$ & $(0.8)$ & $(0.4)$ \\
\hline & & 8.5 & & 8.3 & & & & & 6.2 & & 6.4 \\
\hline & BP & $(0.3)$ & - & $(0.3)$ & - & - & - & - & $(0.3)$ & - & $(0.4)$ \\
\hline \multirow{2}{*}{ Average } & MP & 7.2 & 3.3 & 6.7 & 3.3 & $83 / 35$ & 1.5 & 12.9 & 4.9 & 11.2 & 7.7 \\
\hline & BP & 5.7 & - & 5.4 & 3.0 & $44 /-$ & 2.6 & - & 4.0 & - & 6.8 \\
\hline
\end{tabular}

ww - winter wheat (triticale was used instead of winter wheat in all cases at Vysoké nad Jizerou), alf / clo - alfalfa / clover, sb / pot - sugar beet / potato, rap / mus - rapeseed / mustard, bar - spring barley

* - latter crop was used in Pernolec and Vysoké nad Jizerou 
In Vysoké, the courses of $\mathrm{K}_{\text {avail }}$ were very similar to those in Kostelec. A significant decrease of $\mathrm{K}_{\text {avail }}$ was observed for all treatments between 2010 and $2013(P<0.001) . \mathrm{K}_{\text {avail }}$ of treatment $\mathrm{E}$ was significantly lower than that of other treatments in 2011 and $2012(P<0.05)$. In 2013, however, there were no significant differences among the treatments because of the increase of $\mathrm{K}_{\text {avail }}$ in treatment E.

Trends of $\mathrm{K}_{\text {avail }}$ at sites of higher $\mathrm{K}_{\text {fix }}$ (Prague, Ivanovice and Pernolec) were not so straightfor- ward (Table 6). In 2013, there were no significant differences between treatments at these sites, with exception of significantly higher $\mathrm{K}_{\text {avail }}$ of treatment $\mathrm{F}$ compared to treatments $\mathrm{A}, \mathrm{B}$ and $\mathrm{C}$ in Ivanovice $(P<0.05)$. This treatment in Ivanovice had the highest $\mathrm{K}_{\text {avail }}$ level during the whole experiment.

\section{Soil fixed potassium}

In general, $\mathrm{K}_{\text {fix }}$ differences between treatments were less significant than those of $\mathrm{K}_{\text {avail }}$ (Figure 3). Partially it was due to the higher variability of $\mathrm{K}_{\mathrm{fix}}$

$\mathrm{T}$ a

Potassium uptake at experimental sites $[\mathrm{kg} / \mathrm{ha} / \mathrm{year}]$ by main product (MP), by-product (BP) and total biomass (TB). In brackets - standard deviations.

\begin{tabular}{|c|c|c|c|c|c|c|c|c|c|c|c|}
\hline \multirow{2}{*}{\multicolumn{2}{|c|}{ Site }} & \multicolumn{2}{|c|}{2010} & \multicolumn{5}{|c|}{2011} & \multicolumn{2}{|c|}{2012} & \multirow{2}{*}{$\frac{2013}{w w}$} \\
\hline & & ww & alf / clo* & ww & pea & $\mathrm{sb} /$ pot* & rap / mus & alf / clo* & bar & alf / clo* & \\
\hline \multirow{2}{*}{ Ivanovice } & MP & $\begin{array}{l}19 \\
\text { (3) }\end{array}$ & $\begin{array}{c}57 \\
(11)\end{array}$ & $\begin{array}{l}25 \\
(2)\end{array}$ & $\begin{array}{l}31 \\
(6)\end{array}$ & $\begin{array}{c}66 \\
(16)\end{array}$ & $\begin{array}{c}5 \\
(1)\end{array}$ & $\begin{array}{l}131 \\
(11)\end{array}$ & $\begin{array}{l}13 \\
(2)\end{array}$ & $\begin{array}{l}58 \\
(4)\end{array}$ & $\begin{array}{l}27 \\
(1)\end{array}$ \\
\hline & BP & $\begin{array}{l}18 \\
\text { (1) }\end{array}$ & - & $\begin{array}{l}35 \\
(6)\end{array}$ & $\begin{array}{l}30 \\
(5)\end{array}$ & 154 & $\begin{array}{l}36 \\
(6)\end{array}$ & - & $\begin{array}{l}17 \\
(3)\end{array}$ & - & $\begin{array}{l}41 \\
(3)\end{array}$ \\
\hline \multirow{2}{*}{ Hněvčeves } & MP & $\begin{array}{l}32 \\
(2)\end{array}$ & $\begin{array}{l}70 \\
(3)\end{array}$ & $\begin{array}{l}34 \\
(2)\end{array}$ & $\begin{array}{l}34 \\
(2)\end{array}$ & $\begin{array}{l}192 \\
(22)\end{array}$ & $\begin{array}{c}6 \\
(1)\end{array}$ & $\begin{array}{l}325 \\
(53)\end{array}$ & $\begin{array}{l}23 \\
(2)\end{array}$ & $\begin{array}{l}365 \\
(23)\end{array}$ & $\begin{array}{l}24 \\
(5)\end{array}$ \\
\hline & BP & $\begin{array}{l}67 \\
(5)\end{array}$ & - & $\begin{array}{l}39 \\
(3)\end{array}$ & $\begin{array}{l}32 \\
(4)\end{array}$ & 223 & $\begin{array}{l}43 \\
(5)\end{array}$ & - & $\begin{array}{l}35 \\
(9)\end{array}$ & - & $\begin{array}{c}45 \\
(10)\end{array}$ \\
\hline \multirow{2}{*}{ Kostelec } & MP & $\begin{array}{l}26 \\
(2)\end{array}$ & $\begin{array}{l}87 \\
(4)\end{array}$ & $\begin{array}{l}24 \\
\text { (3) }\end{array}$ & $\begin{array}{l}21 \\
(4)\end{array}$ & $\begin{array}{l}135 \\
(15)\end{array}$ & $\begin{array}{c}5 \\
(1)\end{array}$ & $\begin{array}{l}211 \\
(32)\end{array}$ & $\begin{array}{l}24 \\
(2)\end{array}$ & $\begin{array}{c}220 \\
(8)\end{array}$ & $\begin{array}{l}39 \\
(3)\end{array}$ \\
\hline & $\mathrm{BP}$ & $\begin{array}{l}21 \\
(2)\end{array}$ & - & $\begin{array}{l}10 \\
\text { (1) }\end{array}$ & $\begin{array}{l}19 \\
\text { (4) }\end{array}$ & 248 & $\begin{array}{l}32 \\
(3)\end{array}$ & - & $\begin{array}{l}12 \\
\text { (2) }\end{array}$ & - & $\begin{array}{l}43 \\
(3)\end{array}$ \\
\hline \multirow{2}{*}{ Prague } & MP & $\begin{array}{l}24 \\
(2)\end{array}$ & $\begin{array}{c}67 \\
(10)\end{array}$ & $\begin{array}{l}19 \\
\text { (1) }\end{array}$ & $\begin{array}{l}22 \\
(1)\end{array}$ & $\begin{array}{c}111 \\
(14)\end{array}$ & $\begin{array}{c}6 \\
(1)\end{array}$ & $\begin{array}{l}262 \\
(23)\end{array}$ & $\begin{array}{l}12 \\
(2)\end{array}$ & $\begin{array}{l}202 \\
(24)\end{array}$ & $\begin{array}{l}38 \\
(2)\end{array}$ \\
\hline & $\mathrm{BP}$ & $\begin{array}{l}22 \\
(3)\end{array}$ & - & $\begin{array}{l}11 \\
(2)\end{array}$ & $\begin{array}{l}21 \\
(0)\end{array}$ & 205 & $\begin{array}{l}31 \\
(4)\end{array}$ & - & $\begin{array}{l}12 \\
(2)\end{array}$ & - & $\begin{array}{l}32 \\
(0)\end{array}$ \\
\hline \multirow{2}{*}{ Pernolec } & MP & $\begin{array}{c}7 \\
(2)\end{array}$ & $\begin{array}{l}21 \\
(1)\end{array}$ & $\begin{array}{l}12 \\
\text { (1) }\end{array}$ & $\begin{array}{l}25 \\
(2)\end{array}$ & $\begin{array}{l}113 \\
(3)\end{array}$ & $\begin{array}{l}12 \\
(1)\end{array}$ & $\begin{array}{l}305 \\
(21)\end{array}$ & $\begin{array}{l}18 \\
(2)\end{array}$ & $\begin{array}{c}258 \\
(5)\end{array}$ & $\begin{array}{l}16 \\
\text { (4) }\end{array}$ \\
\hline & BP & $\begin{array}{c}8 \\
(2)\end{array}$ & - & $\begin{array}{l}19 \\
(2)\end{array}$ & $\begin{array}{l}20 \\
(2)\end{array}$ & $102 \mathrm{c}$ & - & - & $\begin{array}{c}11 \\
\text { (1) }\end{array}$ & - & $\begin{array}{l}15 \\
(4)\end{array}$ \\
\hline \multirow{2}{*}{ Vysoké } & MP & $\begin{array}{l}31 \\
(1)\end{array}$ & $\begin{array}{c}70 \\
(15)\end{array}$ & $\begin{array}{l}30 \\
(2)\end{array}$ & $\begin{array}{l}116 \\
(10)\end{array}$ & $\begin{array}{l}189 \\
(11)\end{array}$ & $\begin{array}{l}50 \\
(9)\end{array}$ & $\begin{array}{l}309 \\
(34)\end{array}$ & $\begin{array}{l}28 \\
\text { (1) }\end{array}$ & $\begin{array}{l}248 \\
(21)\end{array}$ & $\begin{array}{l}27 \\
(2)\end{array}$ \\
\hline & BP & $\begin{array}{l}97 \\
(3)\end{array}$ & - & $\begin{array}{l}75 \\
(8)\end{array}$ & - & $171 \mathrm{c}$ & - & $x$ & $\begin{array}{l}31 \\
(2)\end{array}$ & - & $\begin{array}{l}73 \\
(5)\end{array}$ \\
\hline \multirow{3}{*}{ Average } & MP & 23 & 73 & 23 & 30 & 144 & 6 & 259 & 21 & 229 & 29 \\
\hline & BP & 35 & & 29 & 27 & 183 & 36 & & 20 & & 37 \\
\hline & TB & 59 & 73 & 53 & 57 & 327 & 42 & 259 & 41 & 229 & 66 \\
\hline
\end{tabular}

ww - winter wheat (triticale was used instead of winter wheat in all cases at Vysoké nad Jizerou), alf / clo - alfalfa / clover, sb / pot - sugar beet / potato, rap/mus - rapeseed / mustard, bar - spring barley * - latter crop was used in Pernolec and Vysoké nad Jizerou, c - calculated 
contents within replications. In Hněvčeves, Vysoké and Kostelec, alfalfa / clover cropping (treatment E) led to the lowest $\mathrm{K}_{\text {fix }}$, whereas crop sequence with cereals (treatment A) had the highest $\mathrm{K}_{\mathrm{fix}}$. In Hněvčeves, $\mathrm{K}_{\text {fix }}$ decreased markedly in all treatments during the experiment $(P<0.001)$, but this trend was not recorded at other sites.

In Prague and Pernolec, no significant differences of $\mathrm{K}_{\text {fix }}$ were indicated within the whole experimental period. In Ivanovice, significant differences were recognized only in 2012 between $\mathrm{K}_{\text {fix }}$ of treatment $\mathrm{D}$ and treatments $\mathrm{B}, \mathrm{E}$ and $\mathrm{F}(P<0.05)$, but this difference did not appear in other years.
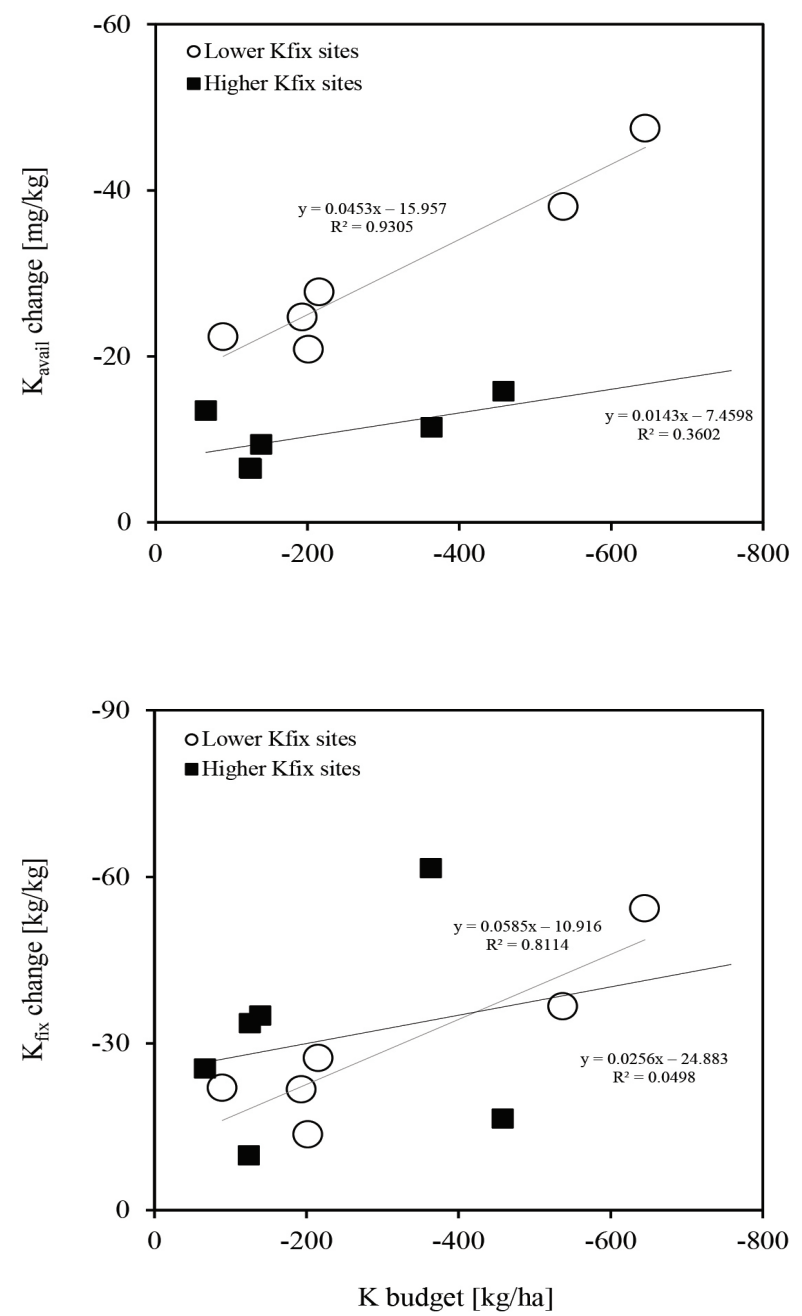

Figure 4. Relation between 3-year budget and change of available and fixed $\mathrm{K}$ contents for lower $\mathrm{K}_{\text {fix }}$ sites (sites of fixed $\mathrm{K}_{\text {fix }}<1,000 \mathrm{mg} \mathrm{K} / \mathrm{kg}$ - Vysoké, Kostelec, Hněvčeves) and higher $\mathrm{K}_{\text {fix }}$ sites $\left(\mathrm{K}_{\text {fix }}>1,000 \mathrm{mg} \mathrm{K} / \mathrm{kg}-\right.$ Ruzyně, Pernolec, Ivanovice)

\section{K budget vs. soil $K$}

A clear trend was found between total $\mathrm{K}$ budget and change in soil $\mathrm{K}_{\text {avail }}$ within 2010 and 2013 for sites of lower $\mathrm{K}_{\text {fix }}$ (Vysoké, Kostelec, Hněvčeves, $P=0.002)$, but no trend was found for sites of higher $\mathrm{K}_{\text {fix }}$ (Figure 4). The same holds also for the change of $\mathrm{K}_{\text {fix }}$, which is significant only for sites of lower $\mathrm{K}_{\mathrm{fix}}(P=0.007)$. As can be seen from the steepness of the $\mathrm{K}_{\text {avail }}$ and $\mathrm{K}_{\text {fix }}$ regression lines, a decrease of $\mathrm{K}_{\text {fix }}$ per a $\mathrm{K}$ budget unit was slightly but insignificantly higher (56\%) than the decrease of $\mathrm{K}_{\text {avail }}(44 \%$ of the decrease of both forms).

\section{DISCUSSION}

\section{K budgets}

The highest $\mathrm{K}$ uptake was recorded for sugar beet, which is known for its high $\mathrm{K}$ demand and high $\mathrm{K}$ content in leaves (on average $4.5 \% \mathrm{~K}$ in dry matter; Neuberg et al. 1980). Potato represents also a large sink of soil $\mathrm{K}$, as majority of this element is present in tubers. Sugar beet can utilize less-available forms of $\mathrm{K}$ (Vaněk et al. 2007). This was confirmed only by $\mathrm{K}_{\text {fix }}$ trend from Kostelec (Figure 3).

In several European long-term fertilisation experiments, annual $\mathrm{K}$ budgets in conventional agricultural systems including sugar beet and potato are in the range from -23 to $-75 \mathrm{~kg} \mathrm{~K} / \mathrm{ha}$ (Blake et al. 1999; Andrist-Rangel et al. 2007). Our K budget for treatment $\mathrm{C}$ is therefore lower; for a 4-year period it is $-124 \mathrm{~kg} \mathrm{~K} / \mathrm{ha} /$ year. It is due to harvesting all by-products, as the intent of the experiment was to keep $\mathrm{K}$ budget as low as possible. Leaves of sugar beet are however usually not harvested in recent agricultural practice. They represent an important $\mathrm{K}$ source and thus compensate high K offtake; Onderka et al. (2001) reported that by post-harvest incorporation of sugar beet leaves, $148 \mathrm{~kg} \mathrm{~K} /$ ha was applied back to soil.

Permanent grasslands are also known for their highly negative $\mathrm{K}$ budget, which is given by high $\mathrm{K}$ concentration in grass/clover biomass (1.2-2.7\% in dry matter; Öborn et al. 2005). Asdal and Bakken (1999) reported that total K budgets were in the range from -228 to $-527 \mathrm{~kg}$ $\mathrm{K} / \mathrm{ha}$ in a 6 -year crop rotation with $50 \%$ of clover 
T a b 1 e 6

Available $\left(\mathrm{K}_{\text {avail }}\right)$ and fixed $\left(\mathrm{K}_{\text {fix }}\right)$ potassium contents $[\mathrm{mg} / \mathrm{kg}]$ during the experiment at sites of higher fixed $\mathrm{K}$. In brackets - standard deviations.

\begin{tabular}{|c|c|c|c|c|c|r|r|r|r|}
\hline \multirow{3}{*}{ Site } & \multirow{2}{*}{ Treatment } & \multicolumn{6}{|c|}{$\mathrm{K}_{\text {avail }}$} & \multicolumn{4}{|c|}{$\mathrm{K}_{\text {fix }}$} \\
\cline { 3 - 10 } & & 2010 & 2011 & 2012 & 2013 & 2010 & 2011 & \multicolumn{1}{|c|}{2012} & 2013 \\
\hline \multirow{5}{*}{ Prague } & $\mathrm{A}$ & $181(7)$ & $186(8)$ & $168(5)$ & $175(7)$ & $991(22)$ & $1,009(20)$ & $1,000(17)$ & $1,032(75)$ \\
& $\mathrm{C}$ & $186(2)$ & $148(4)$ & $165(2)$ & $178(8)$ & $1,015(21)$ & $992(26)$ & $1,014(7)$ & $997(66)$ \\
& $\mathrm{E}$ & $170(10)$ & $156(11)$ & $155(3)$ & $158(23)$ & $994(33)$ & $979(41)$ & $986(23)$ & $1,008(24)$ \\
& $\mathrm{F}$ & $190(13)$ & $176(15)$ & $181(13)$ & $179(9)$ & $1,003(24)$ & $1,007(30)$ & $1,018(22)$ & $1,008(41)$ \\
\hline \multirow{5}{*}{ Ivanovice } & $\mathrm{A}$ & $162(7)$ & $168(8)$ & $160(14)$ & $153(5)$ & $1,075(14)$ & $1,067(14)$ & $1,024(33)$ & $1,047(3)$ \\
& $\mathrm{C}$ & $159(3)$ & $179(13)$ & $156(10)$ & $147(4)$ & $1,087(15)$ & $1,076(18)$ & $1,027(17)$ & $1,057(9)$ \\
& $\mathrm{E}$ & $177(9)$ & $180(18)$ & $188(22)$ & $157(6)$ & $1,069(21)$ & $1,059(21)$ & $992(35)$ & $1,060(48)$ \\
& $\mathrm{F}$ & $189(17)$ & $193(17)$ & $207(28)$ & $180(17)$ & $1,083(32)$ & $1,089(31)$ & $1,013(11)$ & $1,036(5)$ \\
\hline \multirow{5}{*}{ Pernolec } & $\mathrm{A}$ & $96(11)$ & $120(6)$ & $78(8)$ & $90(7)$ & $1,475(96)$ & $1,463(88)$ & $1,451(96)$ & $1,432(64)$ \\
& $\mathrm{C}$ & $85(7)$ & $99(5)$ & $76(7)$ & $81(4)$ & $1,393(175)$ & $1,370(170)$ & $1,347(235)$ & $1,312(167)$ \\
& $\mathrm{E}$ & $109(15)$ & $106(14)$ & $111(33)$ & $94(17)$ & $1,376(209)$ & $1,373(224)$ & $1,371(269)$ & $1,322(289)$ \\
& $\mathrm{F}$ & $105(7)$ & $104(14)$ & $106(11)$ & $86(7)$ & $1,338(177)$ & $1,343(174)$ & $1,347(260)$ & $1,303(248)$ \\
\hline
\end{tabular}

ley; maximum annual $\mathrm{K}$ uptake reached $-175 \mathrm{~kg}$ $\mathrm{K} / \mathrm{ha}$ in the first year of clover. Our results are even higher; in Hněvčeves the K offtake by alfalfa reached $365 \mathrm{~kg} \mathrm{~K} / \mathrm{ha}$ in 2012. Such high offtake is not unrealistic. Øgaard and Krogstad (2005) reported that in non-K-fertilised soils in Norway, annual $\mathrm{K}$ offtake in the first year of grassland exceeded $300 \mathrm{~kg} \mathrm{~K} / \mathrm{ha}$ in some cases.

\section{Soil K pools}

Our results show that $\mathrm{K}_{\text {avail }}$ and $\mathrm{K}_{\text {fix }}$ dynamics depends on the $\mathrm{K}_{\mathrm{fix}}$ level, as majority of significant differences appeared for sites of lower $\mathrm{K}_{\text {fix }}$ content (Vysoké, Kostelec and Hněvčeves). Vopěnka and Macháček (1985) found that $\mathrm{K}_{\text {fix }}$ level have an influence on relation between $\mathrm{K}_{\text {avail }}$ and $\mathrm{K}$ budget. In soils of $\mathrm{K}_{\text {fix }}$ higher than $1200 \mathrm{mg} \mathrm{K} / \mathrm{kg}, \mathrm{K}_{\text {avail }}$ increased even when the budget was negative; in soils of $\mathrm{K}_{\text {fix }}$ lower than $600 \mathrm{mg} \mathrm{K} / \mathrm{kg}, \mathrm{K}_{\text {avail }}$ usually decreased even when the budget was positive. This can explain why almost no significant differences in $\mathrm{K}_{\text {avail }}$ were found in Prague, Ivanovice and Pernolec. Possible differences might be also masked by high $\mathrm{K}_{\text {fix }}$ variability in soils of Prague and Pernolec.

Øgaard and Hansen (2010) studied changes of $\mathrm{K}_{\text {avail }}$ and $\mathrm{K}_{\mathrm{fix}}$ in 6 grassland fertilisation experiments without $\mathrm{K}$ fertilisation. They found large differences between the sites in $\mathrm{K}$ uptake from $\mathrm{K}_{\mathrm{fix}}$. In all sites, $\mathrm{K}_{\text {avail }}$ contributed to total $\mathrm{K}$ uptake (40-183 kg K/ha) by less than $40 \mathrm{~kg} \mathrm{~K} / \mathrm{ha}$ and majority of $\mathrm{K}$ uptake was fulfilled from $\mathrm{K}_{\text {fix }}$. This was observed in Hněvčeves, where $\mathrm{K}_{\text {fix }}$ decreased rapidly, whereas $\mathrm{K}_{\text {avail }}$ remained without significant changes for 5 of 6 treatments. Øgaard and Krogstad (2005) found that $\mathrm{K}$ uptake from $\mathrm{K}_{\text {fix }}$ pool ('interlayer $\mathrm{K}$ ') during 3 years of grass cropping increased with the percentage of clay in the soil consisting of 1 to $34 \%$ clay. For soils containing $>12 \%$ clay, $\mathrm{K}_{\text {fix }}$ release covered $43 \%$ of the $\mathrm{K}$ uptake. In our data, the relation with clay content was not observed, which might be due to a narrower range of clay content in soil.

\section{CONCLUSION}

Negative nutrient budget represents potential risk for intensive agricultural systems. We showed that $\mathrm{K}$ budget can be very negative in case of $\mathrm{K}$ demanding crops. However, by-products are often not harvested in common agricultural praxis, as well as sub-optimal $\mathrm{K}$ application rates replace a part of removed soil $\mathrm{K}$. According to the actual data about $\mathrm{K}$ inputs and outputs in the agricultural 
soils of the Czech Republic, a reasonable estimate of average $\mathrm{K}$ budget is in the range from about $-50 \mathrm{~kg} \mathrm{~K} /$ ha/year (main and by-product harvested) to about $-20 \mathrm{~kg} \mathrm{~K} / \mathrm{ha} /$ year (only main product harvested).

Therefore, based on the linear regressions found, potentially available $\mathrm{K}$ reserve $\left(\mathrm{K}_{\text {avail }}+\mathrm{K}_{\text {fix }}\right)$ decreases annually approximately by from 17 to $32 \mathrm{mg} \mathrm{K} / \mathrm{kg}$ in topsoils with $\mathrm{K}_{\text {fix }}<1,000 \mathrm{mg} \mathrm{K} / \mathrm{kg}$. Approximately half of this depletion originates from $\mathrm{K}_{\mathrm{fix}}$. Therefore, by simple calculation, available $\mathrm{K}$ reserve can be exhausted within 3 to 4 decades in soils of lower $\mathrm{K}_{\text {fix }}$ content. However, such fast 'potassium mining' is not realistic because the plant available pool is continuously replenished from structural $\mathrm{K}$ by weathering of soil minerals.

With respect to K availability, susceptible agricultural soils are soils of low $\mathrm{K}_{\text {fix }}$ used for production of crops with high $\mathrm{K}$ offtake, e.g. forage, potato and sugar beet. In these soils, $\mathrm{K}_{\text {fix }}$ dynamics should be monitored for preventing the risk of decreased $\mathrm{K}$ availability, which can result in yield or quality drop, e.g. in case of a drought stress.

Acknowledgements: The work was supported by the Ministry of Agriculture of the Czech Republic, projects QI91C118 and RO0414.

\section{REFERENCES}

ANDRIST-RANGEL, Y. - EDWARDS, A.C. - HILLIER, S. - ÖBORN, I. 2007. Long-term dynamic of organic and conventional mixed cropping systems as related to management and soil properties. In Agriculture, Ecosystems and Environment, vol. 122, pp. 413-426. DOI: $10.1016 / \mathrm{j}$.agee 2007.02 .007

ANONYMOUS 2013. Zemédělství 2012 [Agriculture 2012]. Prague: Ministry of Agriculture of the Czech Republic. 126 pp. ISBN 978-80-7434-111-3.

ASDAL, A. - BAKKEN, A. 1999. Nutrient balances and yields during conversion to organic farming in two crop rotation systems. In OLESEN, J.E. - ELTUN, R. - GOODING, M.J. - JENSEN, E.S. - KÖPKE U. (Eds.). Designing and testing crop rotations for or ganic farming. Foulum: DARCOF, pp. 125-134.

BLAKE, L. - MERCIK, S. - KOERSCHENS, M. GOULDING, K.W.T. - STEMPEN, S. - WEIGEL, A. - POUlTON, P.R. - POWLSON, D.S. 1999. Potassium content in soil, uptake in plants and the potassium balance in three European long-term field experiments. In Plant and Soil, vol. 216, pp. 1-14. DOI:10.1023/A:1004730023746.
EDMEADES, D.C. - MORTON, J.D. - WALLER, J.E. METHERELL, A.K. - ROBERTS, A.H.C. - CAREY P. 2014. The diagnosis and correction of potassium deficiency in New Zealand pastoral soils: a review. In New Zealand Journal of Agricultural Research, vol. 53, pp. 151-173. DOI:10.1080/00288233.2010. 482954.

GRZEBISZ, W. - DIATTA, J. - HARDTER, R. - CYNA K. 2010. Fertilizer consumption patterns in Central European countries - effect of actual yield development trends in 1986-2005 years - a comparative study of the Czech Republic and Poland. In Journal of Central European Agriculture, vol. 11, no.1, pp.73-82. DOI: 10.5513/JCEA01/11.1.809.

HEJCMAN, M. - BERKOVÁ, M. - KUNZOVÁ, E. 2013. Effect of long-term fertilizer application on yield and concentrations of elements ( $, \mathrm{P}, \mathrm{K}, \mathrm{Ca}, \mathrm{Mg}, \mathrm{As}, \mathrm{Cd}$, $\mathrm{Cu}, \mathrm{Cr}, \mathrm{Fe}, \mathrm{Mn}, \mathrm{Ni}, \mathrm{Pb}, \mathrm{Zn}$ ) in grain of spring barley. In Plant, Soil and Environment, vol. 59, no. 7, pp. 329-334.

HUANG, P.M. 2005. Chemistry of potassium in soils. In TABATABAI, M.A. - SPARKS, D.L. (Eds.). Chemical processes in soils. Madison: SSSA, pp. 227-292. ISBN 0-89118-843-6.

KLÍR, J. - KUNZOVÁ, E. - ČERMÁK, P. 2008. Rámcová metodika výživy rostlin a hnojeni [Frame methodology of plant nutrition and fertilization]. Prague: Crop Research Institute, 48 pp. ISBN 978-8087011-61-4.

MADARAS, M. - KOUBOVÁ, M. - SMATANOVÁ, M. 2014. Long-term effect of low potassium fertilization on its soil fractions. In Plant, Soil and Environment, vol. 60,2014 , no. 8, pp. 358-363.

MARTIN, H.W. - SPARKS, D.L. 1985. On the behaviour of nonexchangeable potassium in soils. In Communications in soil science and plant analyses, vol. 16, pp.133-162. DOI:10.1080/00103628509367593.

MEHLICH, A. 1984. Mehlich-3 soil test extractant: a modification of Mehlich-2 extractant. In Communications in soil science and plant analyses, vol. 15, pp. 1409-1416. DOI:10.1080/00103628409367568.

MORITSUKA, N. - YANAI, J. - KOSAKI, T. 2004. Possible processes releasing nonexchangeable potassium from the rhizosphere of maize. In Plant and Soil, vol. 258, pp. 261-268. DOI:10.1023/B:PLSO.0000016556.79278.7f.

NEUBERG, J. 1980. Komplexni metodika výživy rostlin [The Complex Methodology of Plant Nutrition]. Prague: UVTIZ, $327 \mathrm{pp}$.

ÖBORN, I. -ANDRIST-RANGEL, Y. -ASKEKAARD, M. GRANT, C.A. - WATSON, C.A. - EDWARDS, A.C. 2005. Critical aspects of potassium management in agricultural systems. In Soil Use and Management, vol. 21, no.1, pp. 102-112. DOI:10.1111/j.1475-2743.2005. tb00114.x.

ØGAARD, A.F. - HANSEN, S. 2010. Potassium uptake and requirement in organic grassland farming. In Nutrient Cycling in Agroecosystems, vol. 87, no. 1, pp.137-149. DOI:10.1007/s10705-009-9320-5.

ØGAARD, A.F. - KROGSTAD, T. 2005. Release of interlayer potassium in Norwegian grassland soils. In Journal of Plant Nutrition and Soil Science, vol. 168, pp. 80-88. DOI:10.1002/jpln.200421454. 
ONDERKA, M. - MÍŠA, P. - PROKEŠ, J. - ZIMOLKA, J. - RICHTER, R. - POKORNÝ, E. 2001. Vliv zaorávání chrástu cukrovky na kvalitu sladovnického ječmene [Influence of ploughing in sugar beet leaves on the quality of malt barley]. In Úroda, vol. 47, no.1, pp. 6-7.

REES, G.L. - PETTYGROVEA, G.S. - SOUTHARDA, R.J. 2014. Estimating plant-available potassium in potassium-fixing soils. In Communications in Soil Science and Plant Analysis, vol. 44, pp. 741-748. DOI:10.1080/00103624.2013.748129.

RENGEL, Z. - DAMON, P.M. 2008. Crops and genotypes differ in efficiency of potassium uptake and use. In Physiologia Plantarum, vol. 133, pp. 624-636. DOI:10.1111/j.1399-3054.2008.01079.x.

SCHEFFER, F. - SCHACHTSCHABEL P. 1976. Lehrbuch der Bodenkunde. Stuttgart: Ferdinand Enke Verlag, 394 pp. ISBN 3-432-84779-3.
TORMA, S. 1999. Draslik - dôležitá živina v pôde a v rastline [Importance of potassium for soils and plants]. Bratislava: VÚPOP, 69 p. ISBN 80-85361-51-5.

VANĚK, V. -BALÍK, J. - PAVLÍKOVÁ, D. - TLUSTOŠ, P. 2007. Výživa polních a zahradnich plodin [Nutrition of field and garden plants]. Prague: Profi-Press, pp. 176. ISBN 976-80-86726-25-0.

VOPĚNKA, L. - MACHÁČEK, V. 1985. Bilanční sledování stavu zásobenosti půd draslíkem [Study of the balance of potassium reserve in the soil]. In Rostlinná Výroba, vol. 31, no. 9, pp. 905-912.

ZÖRB, C. - SENBAYRAM, M. - PEITER, E. 2014. Potassium in agriculture - Status and perspectives. In Journal of Plant Physiology, vol. 171, no. 9, pp. 656-669. DOI:10.1016/j.jplph.2013.08.008.

Received: November 18, 2014 